\title{
Microbubble Propulsion, Train-like Assembly and Cargo Transport in an Acoustic Field
}

Jakub Janiak

ETHZ

Alexander Doinikov

ETHZ

Daniel Ahmed ( $\sim$ dahmed@ethz.ch )

ETH Zurich https://orcid.org/0000-0002-0224-5293

\section{Physics Article}

Keywords:

Posted Date: January 20th, 2022

DOI: https://doi.org/10.21203/rs.3.rs-1220770/v1

License: (c) (1) This work is licensed under a Creative Commons Attribution 4.0 International License.

Read Full License 


\title{
Microbubble Propulsion, Train-like Assembly and Cargo Transport in an Acoustic Field
}

\author{
Jakub Janiak, Alexander A. Doinikov, Daniel Ahmed* \\ Acoustics Robotics Systems Lab (ARSL), ETH Zurich, Rüschlikon, CH-8803, Switzerland \\ *Correspondence and requests for materials should be addressed to D.A. \\ (e-mail: dahmed@ethz.ch)
}

\begin{abstract}
Self-assembly, trapping, and transport of microparticles can be pivotal in medicine, lab-on-achip applications, and other fields; however, complex non-Newtonian fluids pose fundamental challenges for these processes. Here, we show that for two glass boundaries set amongst a viscous, shear-thinning gel and separated by a narrow slit $(\sim 20 \mu \mathrm{m})$, incident acoustic waves are concentrated at the interstice, and microbubbles in the surrounding gel exhibit consequent nucleation and other interesting behaviours. When the acoustic field is intermittently activated, microbubbles transform between spherical (off) and ellipsoidal (on) shapes; ellipsoidal microbubbles squeeze into the interstice and become trapped, while spherical ones are pushed out. Upon continuous activation, the ellipsoidal microbubbles execute propulsion driven by Faraday waves superimposed on volume modes developed at their surfaces. When in proximity, they self-assemble into a chain-like microtrain capable of trapping microparticles between its members. Upon deactivation, the microparticles are released, thus simulating a microcargo train.
\end{abstract}


Achieving controlled mobility of microparticles in a biologically-relevant complex fluid medium can create exciting new opportunities in the natural and life sciences and open up biotechniques and biomedical applications, particularly in targeted drug delivery and noninvasive microsurgery. Microparticle transportability has been achieved, generally resorting to i) external-field gradients and ii) instituting nonreciprocal motion within a designed microstructure. The first demonstrated manipulation of dielectric microparticles, using optical tweezers, operated on a tightly-focused gradient of light ${ }^{1,2}$. Later, magnetic ${ }^{3}$, acoustic ${ }^{4-11}$, and electric $^{12}$ field gradients were adopted. Acoustic- ${ }^{13-17}$, electric- ${ }^{18,19}$, magnetic- ${ }^{20-29}$, and light- ${ }^{30}$ based approaches can also initiate propulsion by exploiting nonreciprocity within a microstructure or any appendages anchored to it. However, to date, most manipulation and propulsion of microparticles and microarchitectures has been executed in a simple Newtonian fluid, i.e., water. Although nature's microswimmers such as bacteria ${ }^{31}$, spirochetes $^{32,33}$, and spermatozoa $^{34}$ can navigate effectively in complex fluids and gel-like media, their artificial counterparts find viscous fluids extremely challenging. Only a few synthetic microswimmers have achieved navigation through viscous fluids ${ }^{35}$, such as a magnetic "micro-scallop" that is propelled in non-Newtonian shear-thinning and shear-thickening liquids through reciprocal motion of its appendages ${ }^{36}$. Other magnetic designs have been studied for navigating through bodily fluids such as gelatin ${ }^{37-39}$ and the vitreous humour of the eye $\mathrm{e}^{40}$. In addition, an acoustic vortex beam was recently developed to trap and manipulate microbubbles inside agarose gels that mimic biological tissues ${ }^{41}$.

Herein we report the discovery of various microbubble behaviours in an acoustic field when confined to shallow openings between two glass boundaries in a shear-thinning gel. We observed microbubble nucleation due to intensification of incident acoustic waves at the narrow slit; theoretical development of the pressure field across the glass boundaries supported this acoustic amplification. When the acoustic field was turned off, the microbubbles moved to the sides. Peculiarly, when dormant microbubbles located outside the opening were exposed to ultrasound, they squeezed through the shallow slit, transforming shape from spherical to discoidal just milliseconds prior, and consequently became trapped. Both single and multiple microbubbles were observed to execute controlled propulsion upon activation, the driving mechanism of which we believe stems from superposition of volume and high-amplitude surface modes developed at the microbubble skin. As individual microbubbles approached each other, they self-assembled into a train and travelled in unison at uniform velocity. Surprisingly, when we injected solid microparticles into the surrounding environment, they became trapped between members of the bubble microtrain. Finally, after the train arrived at a destination, the transducer was deactivated and the trapped microparticles released. Our system thus mimics a cargo train at microscale. As most human bodily fluids are shear-thinning, bubble dynamics in such media have implications for medical applications, particularly targeted drug delivery. We envision that acoustically-activated microbubbles can become an exciting platform for drug delivery, such as within the synovial fluid of human joints.

\section{Results}

Experimental setup. Our experimental design incorporates a piezo disc transducer mounted on a glass slide, as shown in the schematic in Fig. 1a (see also Fig. S1). The transducer is 
driven by an electronic function generator to produce elastic Lamb waves on the glass slide. We trigger the transducer's thickness mode at excitation frequencies of $22.3-23 \mathrm{kHz}$ and amplitudes of $20 \mathrm{VPP}$. We apply a viscous, shear-thinning gel to the glass slide at approximately $5 \mathrm{~mm}$ from the transducer. A glass capillary with a circular cross-section is then placed on top of the gel and pressed down until bubbles begin to form and cavitate in the interstice (which occurs at a span of $\sim 20 \mu \mathrm{m}$ between the capillary and the glass), as depicted in Fig. 1b and c. The entire setup was placed on an inverted microscope connected to a highly sensitive, highspeed camera to study the various behaviours of microbubbles (Fig. 1d) within the confines of the narrow aperture.

a
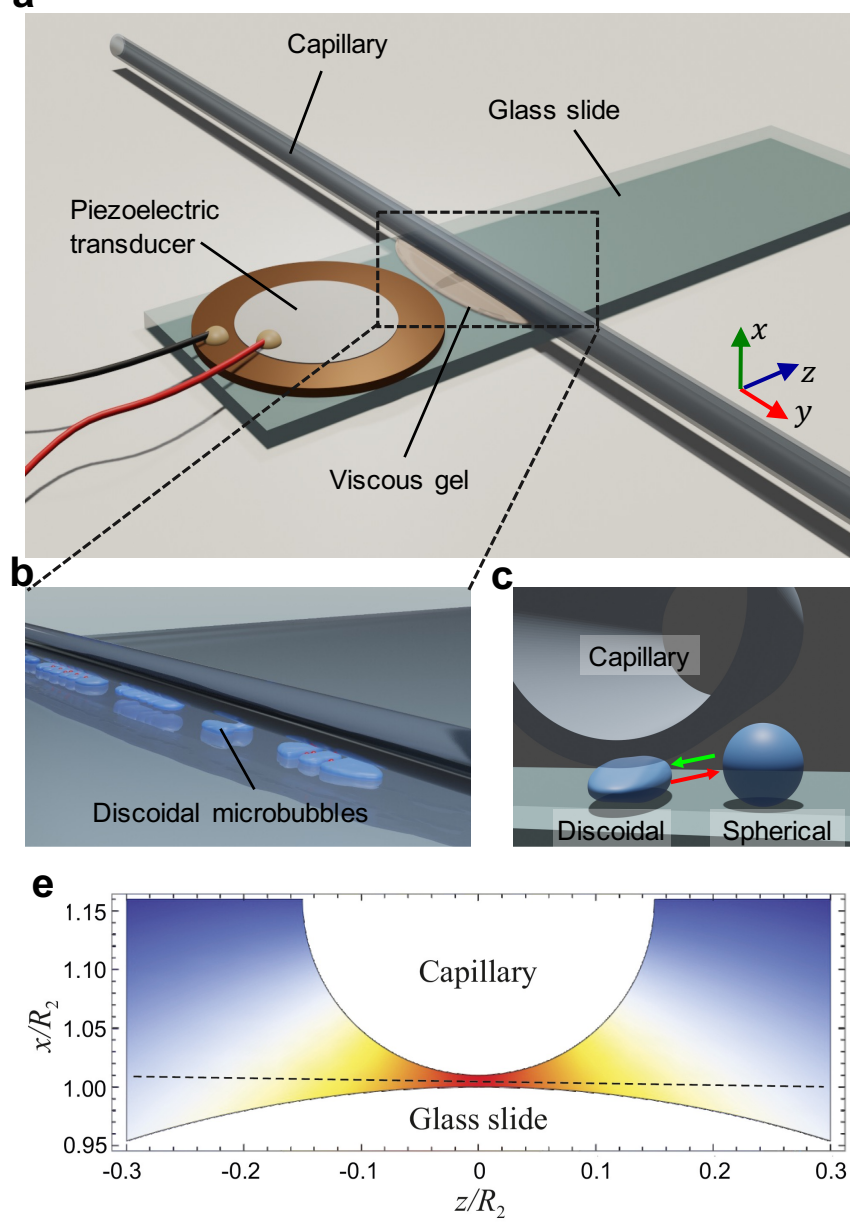

g

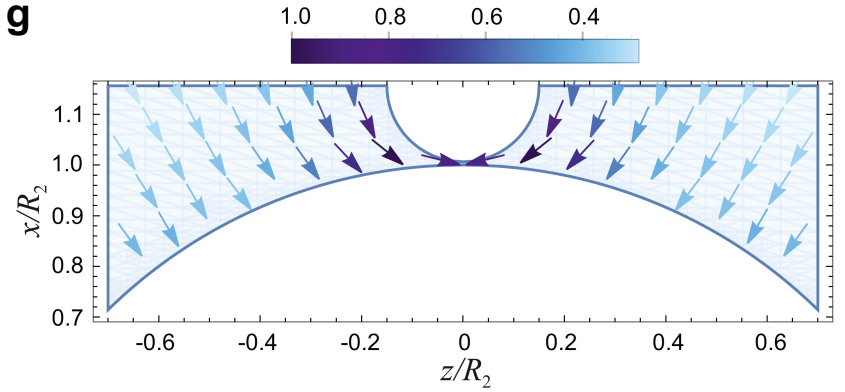

d
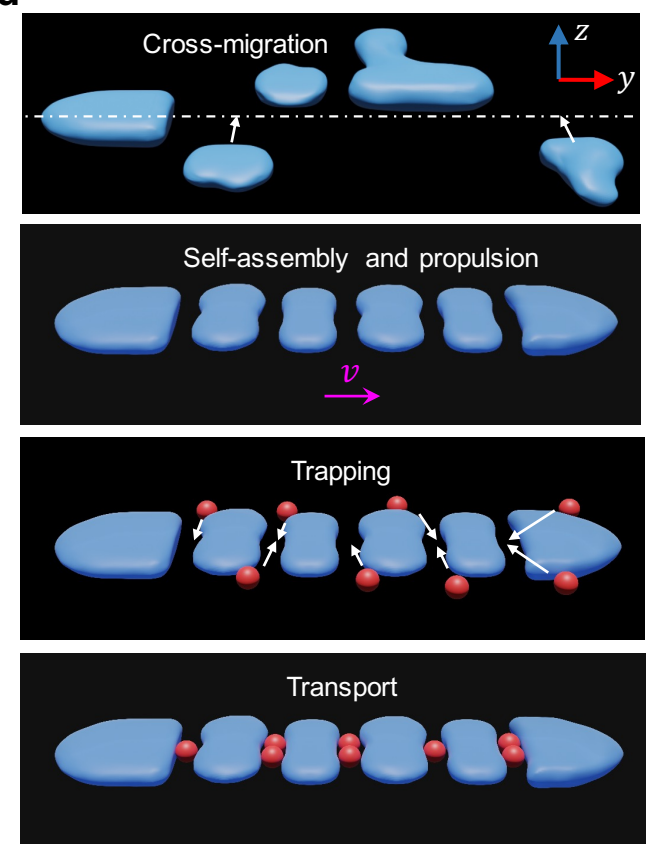

\section{$\mathbf{f}$}

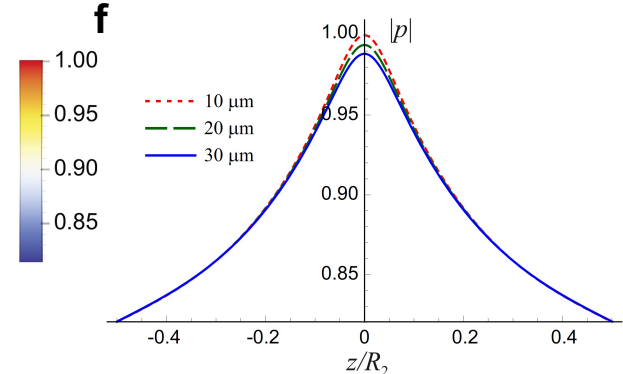

h

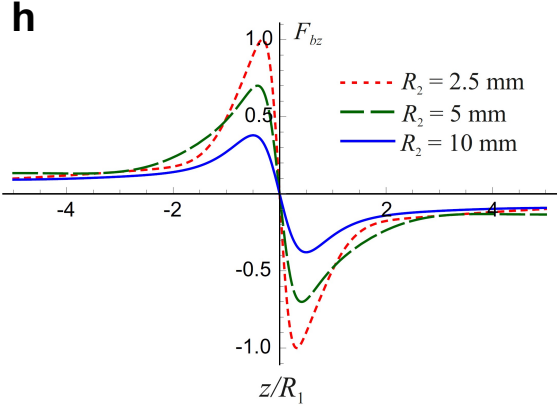

Fig. 1 |Experimental setup and theoretical model. a. Schematic of the experimental setup. A piezo transducer is bonded on a glass slide and, when electrically activated, generates Lamb waves on the slide. A shear-thinning gel is applied 5 to $15 \mathrm{~mm}$ from the transducer. Finally, a 
glass capillary having outer and inner diameters of 1.5 and $1.3 \mathrm{~mm}$, respectively, is positioned on top of the gel, crossing the slide. b. We investigated microbubble behaviours in the shallow region sandwiched between the glass slide and the capillary. c. Microbubbles transformed between spherical and discoidal shapes when the acoustic field was turned on (green arrow) or off (red arrow). d. An illustration of the various behaviors observed: self-assembly, propulsion, trapping, and transport in an acoustically intensified narrow slit. e. Theoretical calculation of the acoustic field generated between two closely spaced cylinders surrounded by a liquid. The radial vibration of the bigger cylinder generates an acoustic field, while the smaller cylinder scatters that field. Distribution of the normalized acoustic pressure magnitude in a plane transverse to the cylinders. The radius of the smaller cylinder is $R_{1}=0.75 \mathrm{~mm}$, the radius of the larger cylinder $R_{2}=5 \mathrm{~mm}$, the vibration frequency $f=23 \mathrm{kHz}$, the distance between the cylinders' surfaces (gap width) $50 \mu \mathrm{m}$ (this value was chosen in order to make the gap between the cylinders visible in the figure), the liquid density $\rho_{0}=1086 \mathrm{~kg} / \mathrm{m}^{3}$, and the speed of sound in the liquid $c=1450 \mathrm{~m} / \mathrm{s}$. f. Variation of the normalized acoustic pressure magnitude along the dashed line depicted in Fig. 1e at three values of the gap width: $10 \mu \mathrm{m}, 20 \mu \mathrm{m}, 30 \mu \mathrm{m} . \mathbf{g}$. Normalized force field experienced by a bubble with radius $R_{0}=25 \mu \mathrm{m}$. The distance between the surfaces of the cylinders is $20 \mu \mathrm{m}$. $\mathbf{h}$. The normalized force component $F_{b z}$, given by Eq. (4), at three values of the radius of the bigger cylinder $R_{2}$ for a bubble located on the dashed line depicted in Fig. 1e.

Modeling of acoustic pressure across a narrow slit. A theoretical model across the narrow slit has been developed that explains the physical mechanism behind the experimental effects we observed (which are described in more detail below). The model approximates the physical situation under study as follows. It is assumed that there are two closely-spaced cylinders, of which the bigger cylinder, with radius $R_{2}$, vibrates radially, generating an acoustic field in the surrounding liquid. Note that the glass slide used in our experiments is approximated as a cylinder whose radius tends to infinity. The smaller cylinder (the capillary), with radius $R_{1}$, scatters the acoustic field generated by the bigger cylinder. The acoustic pressure produced by the cylinders is calculated by $p=-\rho_{0} \partial \varphi / \partial t$, where $\rho_{0}$ is the equilibrium liquid density and $\varphi$ is the potential of the liquid velocity, which obeys the Helmholtz equation ${ }^{42} \nabla^{2} \varphi+k^{2} \varphi=0$ , where $k=\omega / c$ is the wavenumber, $\omega$ is the angular frequency, and $c$ is the speed of sound in the liquid. The calculation gives

$$
p(x, z, t)=i \omega \rho_{0} e^{-i \omega t} \sum_{n=-\infty}^{\infty}\left[a_{n}^{(1)} H_{n}^{(1)}\left(k r_{1}\right) e^{i n \theta_{1}}+a_{n}^{(2)} H_{n}^{(1)}\left(k r_{2}\right) e^{i n \theta_{2}}\right]
$$

where $H_{n}^{(1)}$ is the Hankel function of the first kind, $\left(r_{j}, \theta_{j}\right)$ is the polar coordinates originated at the centre of the $j$ th cylinder $(j=1,2)$, and the coefficients $a_{n}^{(j)}$ are calculated by boundary conditions at the cylinders' surfaces (see Supplementary Section 1.1). The developed theoretical model is valid for any separation distances between cylinders, which allows us to calculate the pressure field at narrow slits.

Figure 1e shows the distribution of $|p|$ in a plane transverse to the cylinders, suggesting an amplification of the acoustic field at the interstice. The plotted values are 
normalized to the maximal pressure value, which occurs at $z=0$, i.e., the midpoint between the cylinders (Fig. 1f). Calculations revealed that, qualitatively, the behaviour of the acoustic pressure remains the same regardless of the cylinders' radii and the distance between them.

Microbubble formation and migration. We first studied the nucleation and cross-migration of microbubbles in a viscous gel through a narrow gap between a capillary and the glass slide. Based on our theoretical model, when an acoustic wave was triggered at a frequency of $23 \mathrm{kHz}$ and voltage of $20 \mathrm{VPP}$, a pressure field formed and was intensified in the space between, which initiated the microbubble nucleation process. As a microbubble oscillates vigorously in this acoustic field, it grows rapidly until reaching the critical size at which it comes into contact with the bounding surfaces of the capillary and the glass slide. The microbubble then conforms to those surfaces, causing it to become discoidal in shape. The unbalanced acoustic pressure distribution within the gap, i.e. the high-pressure gradient along the $+z$ and $-z$-axis, further squeezes the microbubble along the $z$-axis, thus giving rise to an ellipsodal microbubble with its major axis oriented in the $y$-direction. When the acoustic field is turned off, the ellipsoidal microbubbles revert to spherical shape and are squeezed out of the interstice, usually within seconds, migrating to either side of the capillary in the direction of the $z$-axis (see also Supplementary Video 1). It is quite intriguing that when we subsequently re-activated the acoustic field, the microbubbles squeezed back into the slot and reverted to a discoidal shape in a matter of milliseconds.

Knowing the acoustic field produced by the cylinders makes it possible to calculate the acoustic radiation force ${ }^{43}$ that acts on a gas bubble situated beside them (Supplementary Section 1.2). The force is defined by the following equation:

$$
\boldsymbol{F}_{b}=-\frac{4}{3} \pi\left\langle R^{3} \nabla p\right\rangle
$$

where \langle\rangle means the time average, $R$ is the instantaneous bubble radius, which is calculated by the Rayleigh-Plesset equation ${ }^{44,45}$, and $p$ is the acoustic pressure calculated by equation (1). The calculation shows that the radiation force has $x$ and $z$ components, which are given by

$$
\begin{aligned}
& F_{b x}=2 \pi \rho_{0} k R_{0} \operatorname{Re}\left\{\frac{G_{x}(x, z)}{\omega_{0}^{2} / \omega^{2}-1+i \delta}\right\}, \\
& F_{b z}=2 \pi \rho_{0} k R_{0} \operatorname{Re}\left\{\frac{G_{z}(x, z)}{\omega_{0}^{2} / \omega^{2}-1+i \delta}\right\},
\end{aligned}
$$

and lies in the plane transverse to the cylinders. Here, $R_{0}$ is the bubble's equilibrium radius, Re means "the real part of", $\omega_{0}$ is the bubble's resonance frequency, $\delta$ is the damping constant of the bubble's oscillation, $(x, z)$ are the coordinates of the bubble's centre, and the functions $G_{x}(x, z)$ and $G_{z}(x, z)$, which only depend on the acoustic field at the point $(x, z)$, are defined by Supplementary Equations (1.40) and (1.41).

Figures $1 \mathrm{~g}$ and $\mathbf{1 h}$ illustrate predictions of equations (3) and (4), which were calculated with values corresponding to our experimental parameters: $R_{1}=0.75 \mathrm{~mm}$, the distance 
between the cylinders' surfaces $20 \mu \mathrm{m}$, the vibration frequency $f=23 \mathrm{kHz}, \rho_{0}=1086 \mathrm{~kg} / \mathrm{m}^{3}$, $c=1450 \mathrm{~m} / \mathrm{s}$, and the gas inside the bubble is air. Fig. 1g specifically shows the force field experienced by a bubble with radius $R_{0}=25 \mu \mathrm{m}\left(f_{0}=\omega_{0} / 2 \pi=128.7 \mathrm{kHz}\right.$, Eq. 1.31 in Supplementary Information) in the case where $R_{2}=5 \mathrm{~mm}$, while Fig. $\mathbf{1 h}$ depicts the value of the force component $F_{b z}$ for various values of $R_{2}$ assuming that the bubble is sited on the dashed line depicted in Fig. 1e. The force is normalized to the maximal value. It is readily seen that at $z<0, F_{b z}>0$, while at $z>0, F_{b z}<0$. This means that the radiation force causes the bubble to move to the plane $z=0$, where $F_{b z}=0$; that is, it moves to the space between the cylinders and settles there. So long as the acoustic field is on, the position of the bubble at $z=0$ is stable; if the bubble becomes displaced by random perturbations, the radiation force returns it to this position. It should be emphasized that Figs. $\mathbf{1 e}-\mathbf{1 h}$ are strictly concerned with small bubbles, i.e., those with $\omega_{0} / \omega>1$, since our experiments employed such bubbles. In the case depicted, $\omega_{0} / \omega=5.6$.

Discoidal microbubble propulsion. In 1932, Gaines ${ }^{46}$ first noticed a peculiar phenomenon: the erratic behaviour of gas-filled bubbles in a liquid after being irradiated with intense ultrasound ${ }^{46,47}$. This randomly directed, incredibly fast-moving behaviour, dubbed the "dancing bubble" phenomenon, is often difficult to observe, and despite extensive theoretical development, its controlled manipulation has remained difficult to achieve.

With application of an acoustic field, an ellipsoidal microbubble undergoes a volume mode oscillation with amplitude of $\sim 10 \mu \mathrm{m}$ that is superimposed with a Faraday wave at the air/liquid interface, as shown in Fig. 2a and b and Supplementary Videos 2 and 3. In the case of our narrowly-separated capillary and slide setup using a viscous shear-thinning fluid, this resulted in single and multiple microbubbles propelling along the gap in the $y$-direction, as depicted in the image sequences of Fig. 2c (see also Supplementary Video 4). These bubbles attained their terminal velocities within milliseconds and propelled at a remarkable speed of up to $0.6 \mathrm{~mm} / \mathrm{sec}$. The acoustic pressure distribution developed in the interstice, as modelled in Fig. 1f, thus mimics a rail, preventing any sideways deviation of the microbubbles. Initially, we expected that increasing the power applied to the system would cause the bubbles to propel faster; instead, we observed increased power to result in the ellipsoidal microbubbles becoming unstable, behaving erratically or merging with other bubbles.

While we are unable to predict whether microbubbles in this setup will migrate to the left or the right, as they exhibited propulsion in both directions under similar experimental conditions and at similar frequencies and amplitudes of the acoustic field, we are interested in the mechanisms responsible for their propulsion. The translation motion of the microbubbles along the gap suggests that the primary radiation force by the piezo transducer is unlikely to be the driving force. Depending on the resonance frequency of the microbubble, this radiation force will either attract or repel relative to the transducer in the direction of acoustic wave propagation, i.e., along the $z$-axis. However, we observed the ellipsoidal microbubbles to translate along the $y$-axis instead. Furthermore, the spherical microbubbles traversed towards 
the capillary's centre when the acoustic field was activated, regardless of whether they started out above or below the capillary. Based on these observations, it is also unlikely that the primary Bjerknes force is responsible for propulsion. Next, we consider the possible effects arising from a standing wave field. When sound waves propagating along the glass slide impact the glass/air interface, their reflection can result in a standing wavefield in the $z$-direction. Given that the ellipsoidal microbubbles propel along the $y$-axis, this standing wavefield cannot be a source of propulsion. However, a standing acoustic wavefield can also be generated across the glass slide, i.e., along the $y$-axis with the velocity node in the centre. The dispersion equation derived by Doinikov et $\mathrm{al}^{48}$. predicts that, at $23 \mathrm{kHz}$ and given a $20-\mu \mathrm{m}$ narrow slit between the glass slide and capillary, such a standing wave should have a wavelength of 5.6 $\mathrm{cm}$. In view of the fact that the ellipsoidal microbubbles propel in both $+y$ and $-y$ directions, the classical Bjerknes force produced by this standing wave cannot be responsible for the propulsion. The Bjerknes force is directed toward a pressure antinode (velocity node), if the bubble is driven below resonance $\left(\omega<\omega_{0}\right)$ as in our case ${ }^{49,50}$. Note, these results are only valid if the bubble does not undergo strong shape modes.

The acoustic field also excites an elastic wave (Lamb wave) inside the glass slide ${ }^{51}$, which propagates along the $y$-axis. This wave then gives rise to a standing wave in the fluid, which propagates through the intervening fluid layer between the slide and the capillary, with a velocity node developing beneath the centre of the capillary. Since the glass capillary acts as a reflector, the pressure magnitude of this wave across the narrow interstice can be sufficiently high $^{48}$. When a bubble is confined between the slide and the capillary, this wave excites shape oscillation modes (capillary waves) on its surface. The bubble oscillation is inherently nonlinear. This nonlinearity is negligible in a weak driving field. However, in a strong field, this nonlinearity makes surface bubble modes interact with one another and give rise to an additional radiation force on the bubble, which counteracts the classical Bjerknes force (which is directed to the velocity node) and makes the bubble move away from the velocity node. 
a

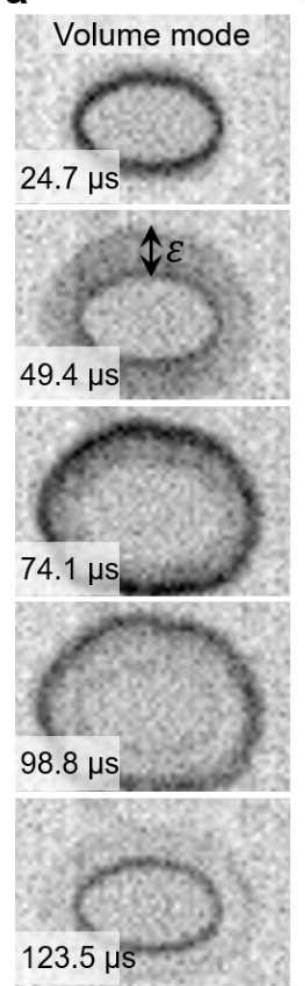

b
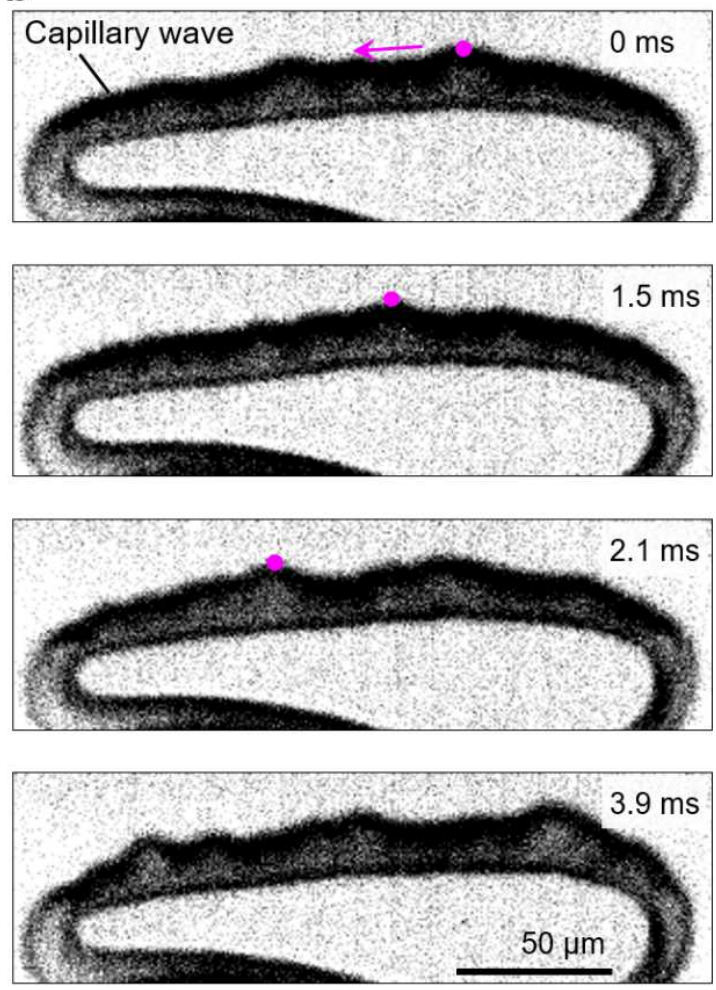

c

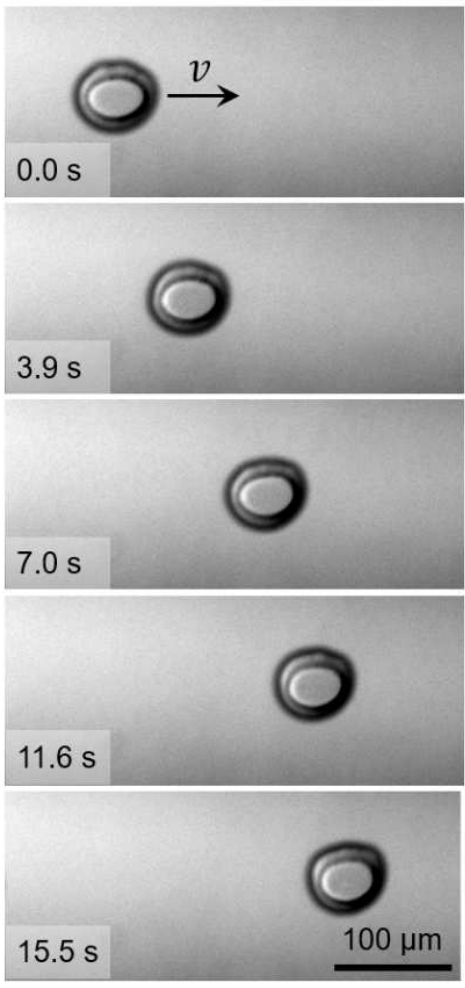

Fig. 2| Oscillation modes and propulsion of ellipsoidal microbubbles in ultrasound. a. Image sequences demonstrating the volume mode oscillation of an ellipsoid microbubble trapped inside the narrow slit. A large oscillation amplitude $(\varepsilon)$ of $\sim 10 \mu \mathrm{m}$ was observed under the bright field microscope. b. A sequence of high-speed images taken at 40,000 frames per second illustrating the Faraday wave on the surface of an elongated microbubble. The capillary wave travels to the left, as indicated by the magenta dot on the crest of the wave. c. Image sequences illustrating the translational motion of a single ellipsoidal microbubble through hydrogel inside a narrow slit when exposed to ultrasound.

Chain-like assembly of microbubbles. When exposed to ultrasound, microbubbles crawled in the $z$-direction and squeezed into the narrow slit, becoming ellipsoidal. The pressure gradient developed across the aperture caused them to accelerate, increasing cross-migration velocity $\left(v_{z}\right)$. The microbubbles subsequently propel themselves along the capillary, translating at different velocities $\left(v_{y}\right)$, but also tend to be drawn together by the secondary Bjerknes force. Specifically, when one microbubble approaches an adjacent microbubble, its velocity $\left(v_{y}\right)$ abruptly increases until the two collide and are required to settle into an equilibrium velocity. Ultimately, this leads the microbubbles to become arranged into a quasi-microtrain, each one mimicking a bogie and the overall length of the structure determined by the number of microbubbles, as illustrated in Fig. 3a and $\mathbf{b}$ and Supplementary Video 5. Among the inner members of the train, a strong repulsive force is manifested, causing a striking gap length of $\sim 10 \mu \mathrm{m}$ between adjacent microbubbles; they do not coalesce. In addition, the inner microbubbles tend to deform from ellipsoidal into almost rectangular shapes and barely oscillate in the $y$-direction, whereas the leading and trailing microbubbles oscillate freely. Despite the apparent repulsion between adjacent microbubbles, a set of bubbles lock themselves together and travel in unison, as illustrated in Fig. 3c. Occasionally, microbubbles 
were observed to eject from the train. Usually, ejection occurred when an interjacent microbubble was significantly smaller than those on either side, as shown by the microbubble tracked with cyan pigment in Fig. $\mathbf{3 b}$.

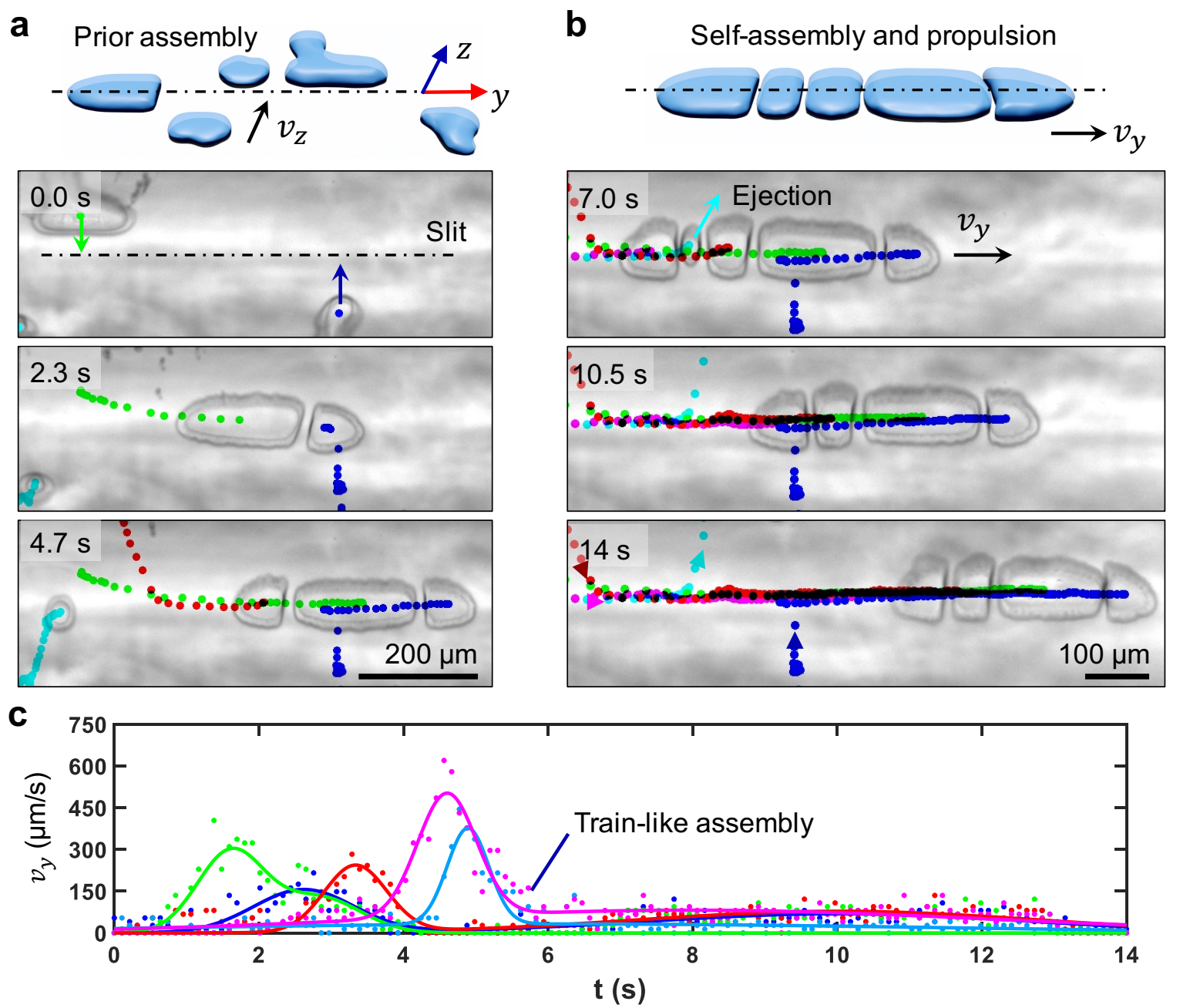

Fig. 3| Self-assembly and propulsion of ellipsoidal microbubbles. a. Schematic and image sequence depicting the microbubbles crawling in the $z$-direction and squeezing into the narrow slit when exposed to ultrasound. These microbubbles then self-assemble into trainlike arrangements. b. Image series demonstrating propulsion of the bubble train, translating left-to-right in the narrow slit. c. Plot of the translational velocities $\left(v_{y}\right)$ of microbubbles moving along the narrow slit in the $y$-direction.

Trapping and transport mechanism. Next, we investigated the trapping and transport behaviour of the microbubble train in an acoustic field. We mixed $10-\mu \mathrm{m}$ polystyrene microbeads with a shear-thinning gel, then sandwiched the resulting solution between the glass slide and capillary. Upon applying an acoustic field, a bubble microtrain was produced. The oscillations of the bubbles subjected the microbeads in the surrounding environment to radiation forces, causing them to be attracted towards the bubbles. As the microparticles approached, they came in contact with the top or bottom surfaces of the microbubbles, then slid left or right along the crosswise centreline until reaching the bubble's equator. The particles then migrated forward or back and became trapped between bubbles, as depicted in Fig. 4a (see also Supplementary Video 6). Moreover, we observed that solid microparticles do not tend to transverse the interstice; rather, they begin to migrate only in the presence of ellipsoidal 
microbubbles, approaching the bubble with a velocity that scales inversely to distance, as shown in Fig. S2. Minimum microbubble oscillations $(\varepsilon \sim 2 \mu \mathrm{m})$ were observed at these regions. No microparticles were observed to be trapped at the top/bottom regions or on the outermost bubbles of the train, which can be attributed to those regions and microbubbles undergoing the largest oscillations, i.e. $\varepsilon \sim 10 \mu \mathrm{m}$.

This behaviour allows for a very reliable trapping mechanism. In addition, the length of the bubble train, i.e., the number of microbubbles, determines the number of microbeads that can be trapped. All told, this represents an exciting novel strategy for transport of microparticles or drugs in extremely viscous liquids or gels.
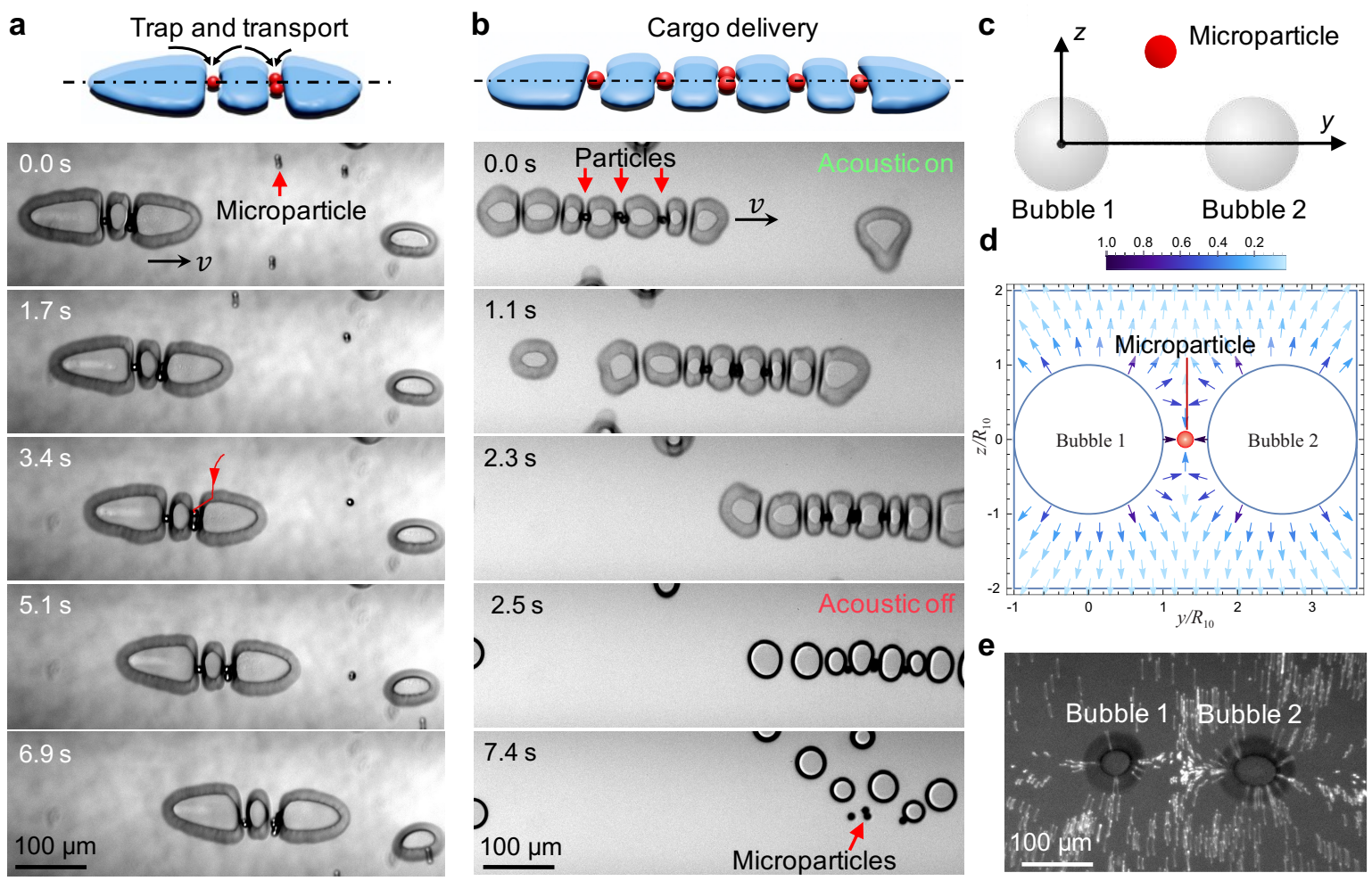

Fig. 4 | Trapping and transport mechanism. The schematic and image sequences demonstrate a. bubble train propulsion and simultaneous trapping of solid particles at the center point between adjacent microbubbles. b. the transport and release of microparticles at 2.5 seconds when the acoustic field is deactivated. Microbubbles emerge from a narrow slit leaving solid particles behind. c. A solid microparticle bounded by two oscillating bubbles in an acoustic field. d. Force field experienced by the microparticle was calculated using the following parameters. The equilibrium bubble radii are $R_{10}=R_{20}=25 \mu \mathrm{m}$. The distance between the bubbles' surfaces is $15 \mu \mathrm{m}$. The bubbles oscillate at $23 \mathrm{kHz}$. The particle, with radius $R_{p}=5 \mu \mathrm{m}$, is made of polystyrene, whose density and sound speed are $\rho_{p}=1060$ $\mathrm{kg} / \mathrm{m}^{3}$ and $c_{p}=2350 \mathrm{~m} / \mathrm{s}$. The sound speed in the liquid is $c=1450 \mathrm{~m} / \mathrm{s}$. The liquid density is $\rho_{0}=1086 \mathrm{~kg} / \mathrm{m}^{3}$. e. Acoustic streamlines created by oscillation of a pair of discoidal microbubbles illustrates the repulsion between them.

Herein we demonstrate the trapping and transport behaviour of the bubble train. The image sequence of Fig. $\mathbf{4 b}$ illustrates the progression of a bubble train and trapped polystyrene microparticles along a predefined path down the long axis of a capillary. When the bubble train 
had travelled $345 \mu \mathrm{m}$, which took 2.3 seconds, we turned the acoustic field off. The discoidalshaped microbubbles ceased oscillating, lacked sufficient energy to remain on the centreline, and so immediately transformed into spherical microbubbles and gradually squeezed out from underneath the capillary. Given the viscous fluid environment, the polystyrene microparticles naturally remained behind at the centreline, and so were separated from the microbubbles (see also Supplementary Video 7).

To explain why solid microparticles are attracted to the space between bubbles, we have calculated the acoustic radiation force that acts on a solid microparticle in the acoustic field generated by two interacting bubbles undergoing axisymmetric oscillation (Supplementary Section 2). To obtain the force, the acoustic field generated by the bubbles is first calculated and then Gor'kov's formula for the force is used ${ }^{52}$. As a result, the following equations for the force components are found:

$$
\begin{aligned}
& F_{p x}=2 \pi \rho_{0} R_{p}^{3}\left[\frac{\omega^{2} f_{1}}{3 c^{2}} H_{x}(x, y)-\frac{f_{2}}{2} I_{x}(x, y)\right], \\
& F_{p y}=2 \pi \rho_{0} R_{p}^{3}\left[\frac{\omega^{2} f_{1}}{3 c^{2}} H_{y}(x, y)-\frac{f_{2}}{2} I_{y}(x, y)\right] .
\end{aligned}
$$

Here $F_{p x}$ is directed along the centreline of the bubbles (Fig. 4c), $F_{p y}$ is perpendicular to the centreline, $f_{1}=1-c^{2} \rho_{0} /\left(c_{p}^{2} \rho_{p}\right), f_{2}=2\left(\rho_{p}-\rho_{0}\right) /\left(2 \rho_{p}+\rho_{0}\right), R_{p}$ is the particle radius, $\rho_{p}$ is the particle density, $c_{p}$ is the speed of sound in the particle, $(x, y)$ are the coordinates of the particle centre, and the functions $H_{x, y}$ and $I_{x, y}$, which only depend on the acoustic field at the point $(x, y)$, are defined by Supplementary Equations (2.35) - (2.38).

Figure 4d shows the normalized force field of two adjacent microbubbles, which was calculated by equations (5) and (6). The calculation was performed at the following parameters. The equilibrium bubble radii are $R_{10}=R_{20}=25 \mu \mathrm{m}$. The distance between the bubbles' surfaces is $15 \mu \mathrm{m}$. The bubbles undergo radial oscillations with a frequency of $23 \mathrm{kHz}$. The particle, with radius $R_{p}=5 \mu \mathrm{m}$, is assumed to be made of polystyrene, whose density and sound speed are $\rho_{p}=1060 \mathrm{~kg} / \mathrm{m}^{3}$ and $c_{p}=2350 \mathrm{~m} / \mathrm{s}$. The sound speed in the liquid is $c=1450$ $\mathrm{m} / \mathrm{s}$ and the liquid density is $\rho_{0}=1086 \mathrm{~kg} / \mathrm{m}^{3}$. The acoustic streamlines created by oscillation of a pair of discoidal microbubbles illustrates the repulsion between them, as illustrated in Fig. 4e (see also Supplementary Video 8). Figure 4d shows that, if the particle gets into the space between the bubbles, the radiation force makes it settle at the midpoint between the bubbles.

It should be noted that in our experiments, we deal with discoidal bubbles. However, there is at present no theory that would allow one to calculate the radiation force produced by such bubbles; the problem is too mathematically complex. Therefore, we have to use theoretical results obtained for spherical bubbles. This approach should be considered an approximation that makes it possible to understand, at least qualitatively, the physical mechanism of the observed effect. 


\section{Discussion}

This article demonstrates the concentration of acoustic waves in a narrow slit and subsequent trapping of microbubbles. We further develop a theoretical model of the pressure field at the interstice. Because the experimental configuration is quite similar to the environment found in joints, the discovered strategy may provide an exciting platform for drug delivery to the synovium of joints, Fig. 5. We expect our study to launch an alternate strategy for micro- and nanoparticle trapping. The observed amplification of sound waves can be applied in slit-like micro- and nanoarchitectures to capture micro- and nanoparticles. We further demonstrate controlled propulsion of an ellipsoidal microbubble, driven by Faraday waves superimposed on its volume mode oscillations. Importantly, while these initial findings are exciting, further research is needed to determine what controls the direction of propulsion. Future work will examine how ellipsoidal microbubbles propel when confined within a slit set in an arbitrary geometry. We also exhibit a new and unique mechanism of trapping microparticles at centerpoints between microbubbles. Future studies will investigate the trapping of particles of different sizes and materials, including glass, metal, and polystyrene. An additional observation of this study is that continuous activation leads the microbubbles to self-assemble into chainlike arrangements that propel in unison. Our findings of self-assembly, trapping, and transport of microparticles in shear-thinning fluid, behaviours ultimately mimicking a cargo train, are of particular interest due to most human bodily fluids being shear-thinning. Studies of bubble dynamics in such a medium might have applications in medicine, especially as a new approach to targeted drug delivery.

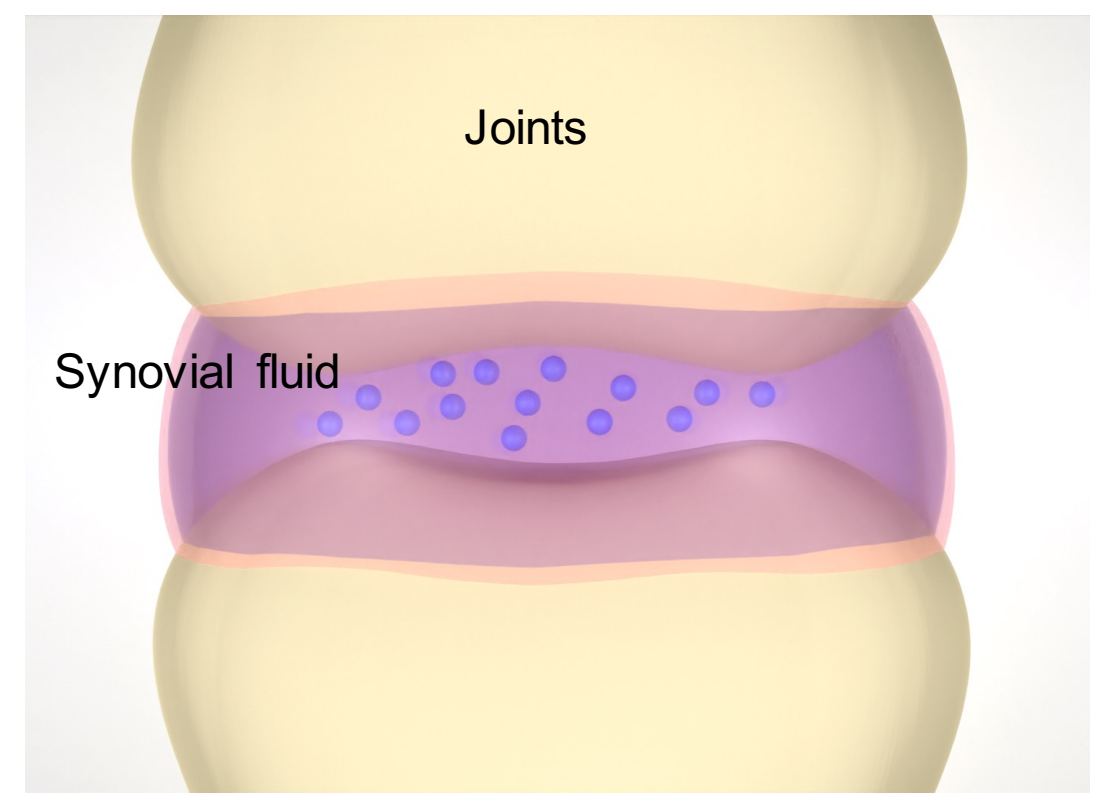

Fig. 5 | A concept for the delivery of drugs to the synovial fluid located within joints. 


\section{Materials and Methods}

Experimental Setup. A piezo transducer is bonded on a glass slide and, when electrically activated, generates Lamb waves on the slide. A viscous gel (K-Y Lubricating Jelly Sterile) is applied to the glass slide. Finally, a glass capillary having an outer diameter of $1.5 \mathrm{~mm}$ is positioned on top of the gel $\sim 5 \mathrm{~mm}$ from the transducer, crossing the slide. The whole setup was positioned on an inverted microscope (ZEISS Axiovert 200M) and experiment results were captured using a high-sensitivity and high-speed camera. The piezoelectric transducer is connected to a function generator capable of creating waves with a peak-to-peak voltage of 20 $\mathrm{V}$ (Tektronix AFG3011C), and is used to generate a square wave with $20 \mathrm{~V}_{\mathrm{PP}}$ and frequencies between 22.3 and $23 \mathrm{kHz}$.

High-speed Image Acquisition. High-speed images of the bubble oscillations were taken at a framerate of $40420 \mathrm{fps}$ with an acoustic excitation frequency of $22.3 \mathrm{kHz}$. Since the bubble oscillation must align with the oscillation of the acoustic field, the period of the oscillation of the bubble should be $45 \mu \mathrm{s}$. The 40420 fps yields a frame length of $25 \mu \mathrm{s}$. Consequently, every 1.8 frames of the video are composed of one oscillation cycle of the bubble, and every 9 frames are composed of 5 cycles. These 9 frames were taken from 5 separate cycles at varying stages of the oscillation. By rearranging the frames chronologically according to the cycle progression, we are able to show 1 oscillation cycle of a bubble in 9 frames, as if it were taken at 202,100 frames per second.

Acknowledgments. This project has received funding from the European Research Council (ERC) under the European Union's Horizon 2020 research and innovation programme grant agreement No 853309 (SONOBOTS) and ETH Research Grant ETH-08 20-1. We would like to thank Prajwal Agrawal and Cornel Dillinger for their helpful discussion.

Author Contributions. J.J. discovered the behavior and performed all the experiments and data analysis with feedback from D.A. A.A.D contributed to the theoretical understanding and developed the analytical solutions. D.A. supervised and designed the experiments and contributed to the scientific presentation and discussion. D.A., A.A.D, and J.J. wrote the manuscript.

Competing Interest Statement. The authors declare no competing financial interests. 


\section{References}

1. Ashkin, A., Dziedzic, J. M., Bjorkholm, J. E. \& Chu, S. Observation of a single-beam gradient force optical trap for dielectric particles. Opt. Lett., 11, 288-290 (1986).

2. Chiou, P. Y., Ohta, A. T. \& Wu, M. C. Massively parallel manipulation of single cells and microparticles using optical images. Nature 436, 370-372 (2005).

3. Wang, X. et al. Intracellular manipulation and measurement with multipole magnetic tweezers. Sci. Robot. 4, eaav6180 (2019).

4. Ding, X. et al. Cell separation using tilted-angle standing surface acoustic waves. Proc. Natl Acad. Sci. USA 111 12992-12997 (2014).

5. Marzo, A. \& Drinkwater, B. W. Holographic acoustic tweezers. Proc. Natl Acad. Sci. USA 116, $84-89$ (2019).

6. Marzo, A. et al. Holographic acoustic elements for manipulation of levitated objects. Nat. Commun. 6, 8661 (2015).

7. Melde, K., Mark, A. G., Qiu, T. \& Fischer, P. Holograms for acoustics. Nature 537, 518522 (2016).

8. Ding, X. et al. On-chip manipulation of single microparticles, cells, and organisms using surface acoustic waves. Proc. Natl Acad. Sci. USA 109, 11105-11109 (2012).

9. Guo, F. et al. Three-dimensional manipulation of single cells using surface acoustic waves. Proc. Natl Acad. Sci. USA 113, 1522-1527 (2016).

10. Caleap, M. \& Drinkwater, B. W. Acoustically trapped colloidal crystals that are reconfigurable in real time. Proc. Natl Acad. Sci. USA 111, 6226-6230 (2014).

11. Dholakia, K., Drinkwater, B. W. \& Ritsch-Marte, M. Comparing acoustic and optical forces for biomedical research. Nat. Rev. Phy.s 2, 480-491 (2020).

12. Wu, M. C. Optoelectronic tweezers. Nat. Photonics 5, 322-324 (2011).

13. Ahmed, D. et al. Selectively manipulable acoustic-powered microswimmers. Sci. Rep. 5, 9744 (2015).

14. Aghakhani, A., Yasa, O., Wrede, P. \& Sitti, M. Acoustically powered surface-slipping mobile microrobots. Proc. Natl Acad. Sci. USA 117, 3469-3477 (2020).

15. Ren, L. et al. 3D steerable, acoustically powered microswimmers for single-particle manipulation. Sci. Adv. (2019) doi:10.1126/sciadv.aax3084.

16. Ahmed, D. et al. Artificial Swimmers Propelled by Acoustically Activated Flagella. Nano Lett. 16, 4968-4974 (2016).

17. Dillinger, C., Nama, N. \& Ahmed, D. Ultrasound-activated ciliary bands for microrobotic systems inspired by starfish. Nat Commun. 12, 6455 (2021).

18. Loget, G. \& Kuhn, A. Electric field-induced chemical locomotion of conducting objects. Nat. Commun. 2, 535 (2011).

19. Wu, Y., Fu, A. \& Yossifon, G. Micromotor-based localized electroporation and gene transfection of mammalian cells. Proc. Natl Acad. Sci. USA 118, (2021).

20. Dreyfus, R. et al. Microscopic artificial swimmers. Nature 437, 862-5 (2005).

21. Ghosh, A. \& Fischer, P. Controlled propulsion of artificial magnetic nanostructured propellers. Nano lett. 9, 2243-5 (2009).

22. Snezhko, A., Belkin, M., Aranson, I. \& Kwok, W.-K. Self-assembled magnetic surface swimmers. Phys. Rev. Lett. 102, 118103 (2009).

23. Zhang, L. et al. Characterizing the swimming properties of artificial bacterial flagella. Nano lett. 9, 3663-7 (2009).

24. Sing, C. E., Schmid, L., Schneider, M. F., Franke, T. \& Alexander-Katz, A. Controlled surface-induced flows from the motion of self-assembled colloidal walkers. Proc. Natl Acad. Sci. USA 107, 535-540 (2010).

25. Jang, B. et al. Undulatory locomotion of magnetic multilink nanoswimmers. Nano Lett. 15, 4829-4833 (2015). 
26. Tasci, T. O., Herson, P. S., Neeves, K. B. \& Marr, D. W. M. Surface-enabled propulsion and control of colloidal microwheels. Nat. Commun. 7, 10225 (2016).

27. Li, J. et al. Magneto-acoustic hybrid nanomotor. Nano Lett. 15, 4814-4821 (2015).

28. Gao, W. et al. Bioinspired helical microswimmers based on vascular plants. Nano Lett. 14, 305-310 (2014).

29. Hu, W., Lum, G. Z., Mastrangeli, M. \& Sitti, M. Small-scale soft-bodied robot with multimodal locomotion. Nature 554, 81-85 (2018).

30. Vutukuri, H. R., Lisicki, M., Lauga, E. \& Vermant, J. Light-switchable propulsion of active particles with reversible interactions. Nat. Commun. 11, 2628 (2020).

31. Sachs, G., Weeks, D. L., Melchers, K. \& Scott, D. R. The Gastric Biology of Helicobacter pylori. Annu. Rev. Physiol. 65, 349-369 (2003).

32. Li, C., Motaleb, A., Sal, M., Goldstein, S. F. \& Charon, N. W. Spirochete periplasmic flagella and motility. J. Mol. Microbiol. 2, 345-354 (2000).

33. Li, C., Motaleb, A., Sal, M., Goldstein, S. \& Charon, N. Spirochete periplasmic flagella and motility. J. Mol. Microbiol. 2, 345-54 (2000).

34. Tung, C. et al. Fluid viscoelasticity promotes collective swimming of sperm. Sci. Rep. 7, 3152 (2017).

35. Kroo, L. A., Binagia, J. P., Eckman, N., Prakash, M. \& Shaqfeh, E. S. G. A Swimming Rheometer: Self-propulsion of a freely-suspended swimmer enabled by viscoelastic normal stresses. arXiv:2111.10515 (2021).

36. Qiu, T. et al. Swimming by reciprocal motion at low Reynolds number. Nat. Commun. 5, 5119 (2014).

37. Ullrich, F. et al. Swimming characteristics of helical microrobots in fibrous environments. in 2016 6th IEEE International Conference on Biomedical Robotics and Biomechatronics (BioRob) 470-475 (2016).

38. Ullrich, F. et al. Swimming characteristics of helical microrobots in fibrous environments. in 2016 th IEEE International Conference on Biomedical Robotics and Biomechatronics (BioRob) 470-475 (2016). doi:10.1109/BIOROB.2016.7523671.

39. Walker, D., Käsdorf, B. T., Jeong, H.-H., Lieleg, O. \& Fischer, P. Enzymatically active biomimetic micropropellers for the penetration of mucin gels. Science Advances (2015) doi:10.1126/sciadv.1500501.

40. Wu, Z. et al. A swarm of slippery micropropellers penetrates the vitreous body of the eye. Sci. Adv. (2018) doi:10.1126/sciadv.aat4388.

41. Acoustic trapping of microbubbles in complex environments and controlled payload release Proc. Natl Acad. Sci. USA 117 (27) 15490-15496 (2020).

42. Landau, L. D. \& Lifshitz, E. M. Fluid Mechanics (Pergamon Press, Oxford, 1987).

43. Doinikov, A. A. Acoustic radiation forces: Classical theory and recent advances. Recent research developments in acoustics 1, 39-67 (2003).

44. Rayleigh, Lord. On the pressure developed in a liquid during the collapse of a spherical cavity. Phil. Mag. 34, 94-98 (1917).

45. Plesset, M. S. The Dynamics of Cavitation Bubbles. J. Appl. Mech. 16, 277-282 (2021).

46. Gaines, N. A magnetostriction oscillator producing intense audible sound and some effects obtained. J. Appl. Phys. 3, 209-229 (1932).

47. Doinikov, A. A. Translational motion of a bubble undergoing shape oscillations. J. Fluid Mech. 501, 1-24 (2004).

48. Doinikov, A. A., Thibault, P. \& Marmottant, P. Acoustic streaming in a microfluidic channel with a reflector: Case of a standing wave generated by two counterpropagating leaky surface waves. Phys. Rev. E 96, 13101 (2017).

49. Eller, A. Force on a bubble in a standing acoustic wave. J. Acoust. Soc. 43, 170-171 (1968). 
50. Crum, L. A. \& Eller, A. I. Motion of bubbles in a stationary sound field. J. Acoust. Soc. 48, 181-189 (1970).

51. Viktorov, I. A. Rayleigh and Lamb Waves: Physical Theory and Applications (Plenum Press, New York, 1967).

52. Gor'kov, L. P. On the forces acting on a small particle in an acoustical field in an ideal fluid. in Sov. Phys. Dokl. vol. $6773-775$ (1962). 


\section{Supplementary Files}

This is a list of supplementary files associated with this preprint. Click to download.

- SupplementaryVideo3.avi

- Supplementaryinformation.pdf

- SupplementaryVideo2.avi

- Supplementaryvideo1.avi

- SupplementaryVideo6.avi

- SupplementaryVideo5.avi

- SupplementaryVideo4.avi

- SupplementaryVideo7.avi

- SupplementaryVideo8.avi 